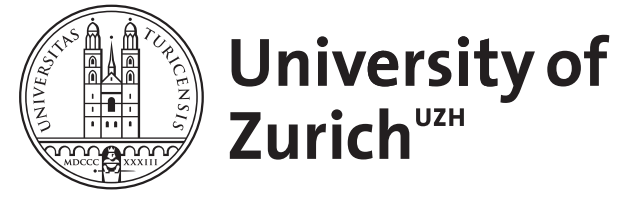

Zurich Open Repository and Archive

University of Zurich

University Library

Strickhofstrasse 39

CH-8057 Zurich

www.zora.uzh.ch

Year: 2020

\title{
Sports Psychiatry: Exercise and postpartum depression
}

Imboden, Christian ; Claussen, Malte Christian

DOI: https://doi.org/10.34045/sems/2020/43

Posted at the Zurich Open Repository and Archive, University of Zurich

ZORA URL: https://doi.org/10.5167/uzh-211809

Journal Article

Published Version

Originally published at:

Imboden, Christian; Claussen, Malte Christian (2020). Sports Psychiatry: Exercise and postpartum depression. SEMS-journal, 68(4):30-31.

DOI: https://doi.org/10.34045/sems/2020/43 


\title{
Sports Psychiatry: Exercise and postpartum depression
}

\author{
Imboden Christian', Claussen Malte Christian'1,2,3 \\ 'Private Clinic Wyss AG, Münchenbuchsee, Switzerland \\ 2 Department of Psychiatry, Psychotherapy and Psychosomatics, University Hospital of Psychiatry Zurich, Zurich, Switzerland \\ ${ }^{3}$ Psychiatric Services Grisons, Chur, Switzerland
}

Postpartum depression (PPD) is a common psychiatric disorder in the peripartum period affecting approximately $8 \%$ of European women without prior psychiatric history [1] and even more with pre-existing depressive disorders. Up to $70 \%$ of new mothers develop mild depressive symptoms called "baby-blues" which include weepiness, sadness and mood lability. Those symptoms usually peak between 2 and 5 days post-delivery and abate spontaneously within days up to 2 weeks [2]. In contrast to "baby blues" postpartum depression can have a serious impact on functioning and even lead to suicidality rendering it highly important to detect the disorder as early as possible. The Edinburgh Postnatal Depression Scale (EPDS) [3] is widely used to screen for postpartum depression and should be applied to all new mothers who develop depressive symptoms such as loss of interest, hopelessness and depressive feelings. Several risk factors for postpartum depression have been identified: Prenatal depression poses the highest risk, followed by low self-esteem, childcare stress, prenatal anxiety, life stress and lack of social support [4].

Postpartum depression is a treatable condition, however, it has to be distinguished from bipolar disorder and postpartum psychosis, which require different treatment strategies [2]. Mild to moderate forms can be treated well with psychosocial interventions and psychotherapy, its severe forms require pharmacological treatment with selective serotonine reuptake-inhibitors (SSRI). SSRI-treatment usually is considered to be compatible with breast-feeding of healthy infants [5] but may still have adverse effects on the baby such as irritability, poor weight gain and sedation [6]. Therefore, if non-pharmacological treatments are not effective enough acceptability of an antidepressant might pose a problem for mothers.

Physical activity (PA) has proven to effectively prevent the onset of depression in the general population with a risk reduction of approximately 17\% [7] and might even be a viable treatment strategy in mild to moderate depression $[8,9]$. Therefore, PA might also have beneficial effects on PPD. However, Promoting PA has not yet been established as a standard procedure in psychiatric care as the authors addressed in their position paper of the Swiss Society of Sports Psychiatry and Psychotherapy [10].

\section{Physical activity during pregnancy and PPD}

A study with $\mathrm{n}=597$ German mothers has found a significant decrease of PA from the $20^{\text {th }}$ to the $32^{\text {nd }}$ gestational week. Moreover, a higher decrease of PA during pregnancy was associated with significantly higher depression- (EPDS) and anxiety-scores [11]. A Spanish study randomized $n=129$ pregnant women at the $20^{\text {th }}$ gestational week to either 12 weeks water-based exercise or no intervention. Exercise took place three times per week for one hour. They found a significant reduction of PPD-scores in the exercise group. The effect was even more significant in overweight to obese women [12]. On the other hand, a study conducted with Brasilian women, randomizing $\mathrm{n}=639$ women between 16 and 20 weeks of gestation to either 16 weeks of supervised combined (aerobic and strength) exercise or no intervention only found significant effects of exercise on the reduction of depression scores ante- but not postnatally. However, adherence was very low, reaching only $42.7 \%$ of women. Therefore, the results have to be interpreted with caution [13]. A recent meta-analysis on 6 interventional studies found a significant preventive effect on postpartum depression for exercise interventions during pregnancy [14].

\section{Physical activity for PPD}

In an English trial $n=94$ women with PPD during the first 6 months after delivery were randomized to either a structured telephone- and face to face-coaching or treatment as usual for 6 months. The goal was to achieve at least 30 minutes of moderate intensity exercise on three days per week. After 6 months the exercise group showed increased PA and significantly lower EPDS-scores as well as higher social support than controls. Therefore, exercise had beneficial effects on PPD which even extended to social support [15], a wellknown risk factor of PPD [4]. In a meta-analysis conducted over 16 randomized controlled trials a positive effect of exercise was found in postpartum depression scores despite the fact, that the methodology of included studies varied greatly: Exercise programs took place from 6 weeks up to 12 months and varied from once a week to 5 times a week. In most studies aerobic exercise of light to medium intensity was applied. Exercise showed a stronger effect in women already diagnosed with PPD (9 studies) and if exercise took place under supervised conditions [16]. 


\section{Conclusions}

There is growing evidence that physical activity might protect from PPD and even be helpful during its treatment. However, there is still a need for high-quality studies on exercise as treatment for PPD to further strengthen those findings. PA could serve as a non-pharmacological treatment-alternative without adverse effects and high acceptability. Since physical activity has shown to have positive effects on a variety of pregnancy-related conditions such as gestational weight-gain and diabetes [17] there is a broad evidence-base supporting the promotion physical activity during pregnancy. Taken together, keeping up or developing regular exercise routines during pregnancy or after delivery seems to be highly advisable to reduce the risk of developing postpartum depression in all women. From a public health-perspective there is a need to develop accessible programs for pregnant women and mothers of newly born children as well as to rise awareness of the association of PA and postpartum depression.

\section{Corresponding author}

Dr. med. Christian Imboden EMBA, Private Clinic Wyss AG, Münchenbuchsee, Switzerland christian.imboden@pkwyss.ch

\section{References}

1. Shorey S, Chee CYI, Ng ED, Chan YH, Tam WWS, Chong YS. Prevalence and incidence of postpartum depression among healthy mothers: A systematic review and meta-analysis. J Psychiatr Res. 2018;104:235-48.

2. Stewart DE, Vigod S. Postpartum Depression. N Engl J Med. 2016;375(22):2177-86.

3. Schaper AM, Rooney BL, Kay NR, Silva PD. Use of the Edinburgh Postnatal Depression Scale to identify postpartum depression in a clinical setting. J Reprod Med. 1994;39(8):620-4.

4. Beck CT. Predictors of postpartum depression: an update. Nurs Res. 2001;50(5):275-85.

5. Kim DR, Epperson CN, Weiss AR, Wisner KL. Pharmacotherapy of postpartum depression: an update. Expert Opin Pharmacother. 2014;15(9):1223-34.

6. Pearlstein T, Howard M, Salisbury A, Zlotnick C. Postpartum depression. Am J Obstet Gynecol. 2009;200(4):357-64.

7. Schuch FB, Vancampfort D, Firth J, Rosenbaum S, Ward PB, Silva ES, et al. Physical Activity and Incident Depression: A Meta-Analysis of Prospective Cohort Studies. Am J Psychiatry. 2018:appiajp201817111194.

8. Kvam S, Kleppe CL, Nordhus IH, Hovland A. Exercise as a treatment for depression: A meta-analysis. Journal of affective disorders. 2016;202:67-86.

9. Morres ID, Hatzigeorgiadis A, Stathi A, Comoutos N, Arpin-Cribbie C, Krommidas C, et al. Aerobic exercise for adult patients with major depressive disorder in mental health services: A systematic review and meta-analysis. Depress Anxiety. 2019;36(1):39-53.

10. Imboden C, Claussen MC, Gerber M, Gonzalez Hofmann C, Hemmeter U, E S. Swiss Society for Sports Psychiatry and Psychotherapy SSSPP. SSSPP Position paper: Physical activity and mental health. Swiss Sports \& Exercise Medicine. 2020;68(3):14-18.

11. Hassdenteufel K, Feisst M, Brusniak K, Lingenfelder K, Matthies LM, Wallwiener M, et al. Reduction in physical activity significantly increases depression and anxiety in the perinatal period: a longitudinal study based on a self-report digital assessment tool. Arch Gynecol Obstet. 2020;302(1):53-64

12. Aguilar-Cordero MJ, Sanchez-Garcia JC, Rodriguez-Blanque R, Sanchez-Lopez AM, Mur-Villar N. Moderate Physical Activity in an Aquatic Environment During Pregnancy (SWEP Study) and Its Influence in Preventing Postpartum Depression. J Am Psychiatr Nurses Assoc. 2019;25(2):112-21.

13. Coll CVN, Domingues MR, Stein A, da Silva BGC, Bassani DG, Hartwig FP, et al. Efficacy of Regular Exercise During Pregnancy on the Prevention of Postpartum Depression: The PAMELA Randomized Clinical Trial. JAMA Netw Open. 2019;2(1):e186861.

14. Nakamura A, van der Waerden J, Melchior M, Bolze C, El-Khoury F, Pryor L. Physical activity during pregnancy and postpartum depression: Systematic review and meta-analysis. J Affect Disord. 2019;246:29-41.

15. Daley AJ, Blamey RV, Jolly K, Roalfe AK, Turner KM, Coleman S, et al. A pragmatic randomized controlled trial to evaluate the effectiveness of a facilitated exercise intervention as a treatment for postnatal depression: the PAM-PeRS trial. Psychol Med. 2015;45(11):2413-25.

16. McCurdy AP, Boulé NG, Sivak A, Davenport MH. Effects of Exercise on Mild-to-Moderate Depressive Symptoms in the Postpartum Period: A Meta-analysis. Obstet Gynecol. 2017;129(6):1087-97.

17. Dipietro L, Evenson KR, Bloodgood B, Sprow K, Troiano RP, Piercy KL, et al. Benefits of Physical Activity during Pregnancy and Postpartum: An Umbrella Review. Med Sci Sports Exerc. 2019;51(6):1292302 . 